\title{
The perils of intersectionality: racial and sexual harassment in medicine
}

\author{
Sherita Hill Golden \\ Department of Medicine, Johns Hopkins University School of Medicine, Baltimore, Maryland, USA.
}

It was early evening in the fall of 1994 as I began my long call. I met the daughter of the next patient I was admitting while she was waiting for her mother to return from interventional radiology. I recall that evening like it was yesterday. I was adorned in a clean, short white coat over a green blouse and navy blue slacks. My stethoscope was in one pocket and my medicine manual in the other. After being informed that my patient had returned, I went to her room. Before I could introduce myself as Dr. Hill, she said, 'Oh, thank goodness you are here. Are you the TV lady? I have been waiting for someone to come up here to collect the money so I can get my TV turned on!' While I was frozen in place trying to process what I had just heard, her daughter, exasperated and embarrassed, exclaimed, 'Heavens Mother! That is your doctor!'

That occasion would, unfortunately, not be the last time during my intern year that I would be mistaken for the TV lady, dietary staff, or other non-physician support staff. Each time it occurred, I felt degraded and demoralized. I never shared these feelings with anyone because it seemed too sensitive and painful to articulate. My turmoil was further heightened by the high regard and respect I had for the important role of our support staff in caring for our patients. I was afraid that disclosing my frustration and pain at being assumed to be non-physician staff would alienate me from the individuals who were part of my strongest support system on the wards during my internship. I also could not sort out whether these experiences were occurring due to my race or to my sex.

A 2018 National Academies of Science, Engineering, and Medicine consensus study uncovered the alarming frequency of gender harassment, unwanted sexual attention, and sexual coercion in academic science and medicine (1). More than 50\% of women faculty and staff report having been harassed at their academic institutions (1), with gender harassment being the most common form. Women students, trainees, and faculty in academic medical centers experience sexual harassment not only by their peers and supervisors but also, by patients and patients' families. For women who are underrepresented in medicine (URM), there is the added complexity of intersectionality - wherein both race and gender create overlapping and interdependent systems of discrimination (2).

Though limited research in this area exists, the National Academies report highlights the current literature on intersectionality. Women of color are more likely to feel unsafe in the academic environment because of their race compared to men of color, white men, or white women (2). They are also more likely to experience verbal racial harassment (compared with men of color and white men and women) and equally likely as white women to experience verbal sexual harassment (2). In addition, black women, Asian American women, and Latinas report overall sexual harassment less frequently than white women (3).

The experience of racial and sexual harassment has adverse effects on mental health and job satisfaction in minority women. All women, including women of color, who experience sexual harassment report more symptoms of depression, stress and anxiety, and impaired psychological well-being (1). Among Latina women, sexual harassment was more distressing when occurring simultaneously with other types (e.g., race-based) of harassment in the workplace (4), and more severe harassment was associated with lower job satisfaction; leading to job withdrawal, and increased depressive, anx-

Conflict of interest: The author has declared that no conflict of interest exists.

iety, and somatic symptoms (1). Among black women, race of the perpetrator is a powerful predictor of sexual harassment appraisal, and cross-racial harassment is perceived to be more offensive, frightening, and disturbing than intraracial harassment (5). Additional research is needed to further elucidate the impact of intersectionality on health and job satisfaction and productivity among URM women.

Overall, sexual harassment is perceived by women to be more severe in work and education environments that tolerate sexual, racial, and sexual-racial harassment. So how do we begin to change this dynamic? A key recommendation in the National Academies report is to change climate and culture to prevent and effectively address sexual harassment (1).

\section{The value proposition for diversity across disciplines}

As recently reviewed, diverse teams make better decisions and have improved financial performance, as diversity "facilitates friction that enhances deliberation and upends conformity (6)." A diverse healthcare workforce helps to reduce or eliminate health disparities (7). In the education setting, a large-scale study showed that student performance improved most when there was greater management diversity and diversity that more closely reflected the composition of the student population (8), which emphasizes the importance of leadership in setting the tone and allocating resources. Diverse teams are also more productive. A prodiversity climate at a major national U.S. retailer was associated with higher sales production for black and Hispanic employees (6). In the field of science, ethnicity had strongest correlation with the scientific impact of papers, regardless of year of publication, number of authors per paper, and number of collaborations per scientist; ethnic diversity resulted in $10.63 \%$ impact gain for papers and $47.67 \%$ impact gain for scientists 
Table 1. Recommendations for addressing sexual harassment and improving diversity in academic medicine

\author{
National Academies of Medicine, Science, and Engineering Recommendations (1) \\ Create diverse, inclusive, and respectful environments and integrate that into policies \\ and procedures \\ Diffuse the hierarchical and dependent relationship between trainees and faculty \\ Provide support for targets of harassment \\ Improve transparency and accountability \\ Strive for strong and diverse leadership \\ Make the entire academic community responsible for reducing and preventing sexual \\ harassment
}

\section{Other Recommendations}

Train members of the medical and scientific communities how to respond to sexual and racial harassment in real-time

Implement unconscious bias training and bias reduction interventions

Support programs to increase URM physician-scientists, including pipeline programs for high school students, undergraduates, graduate and medical students, and post-doctora fellows as well as URM Junior faculty mentorship programs
(9). From a business perspective, boards with greater gender and racial diversity engaged in less risky investment behavior, fulfilled monitoring responsibilities more often (resulting in higher accounting returns), and had companies with greater innovation and positive return on assets and investments (6). Importantly, diversification must extend beyond tokenism - in one business study, the proportion of women in top management had to exceed $20 \%$ for the company to experience an increase in innovation revenues (6).

\section{Status of URM women in academic medicine}

There remains a dearth of URM individuals in science and medicine, particularly in the academic setting, with the lowest figures being for URM women. Only 6\% of advanced degrees in science and engineering were awarded to URM women (10), and nonwhite women are underrepresented in the biomedical workforce among research project grants (RPG) and R01-equivalent NIH awardees (11). From 2000 to 2010 , blacks comprised only $1.5 \%$ of the R01 applicant pool (12), and Hispanics and blacks remain underrepresented in academic medicine across more than 15 specialties (13). The pipeline is also shallow - the cumulative percentage of Medical Scientist Training Program students from URM backgrounds from 1975 to 2015 is < 5\% (4\% Hispanic, 3\% Black, 0.3\% Native American; and 0.2\% Pacific) (14).

\section{A call to action - what we can do}

The National Academies report made several recommendations (Table 1), including the critical need for academic institutions to create policies and procedures to pro- mote diverse, inclusive, and respectful environments. We need to foster an open environment that minimizes the negative consequences of frictions that are inevitable with culture change and recognize that progress from diversity is maximized when reflected in top leadership positions and extends beyond tokenism (6). Policy and programmatic interventions at the University of California, San Diego to improve faculty equity, diversity, culture, and climate over a 10-year period resulted in an increase in tenure and tenure-track among women and URMs faculty, a decrease in gender pay disparity, and a decrease in inappropriate behavior by faculty (15). We can also teach our bystander colleagues how to respond inappropriate racial and gender harassment. The Johns Hopkins Department of Medicine hosted a symposium to assist healthcare providers at all levels in supporting and advocating for colleagues who are victims of patient discrimination; a model that can be extended to other discrimination scenarios (16). Wheeler et al. recommend twelve tips for responding to microaggressions and overt discrimination from patients (17), which women in academic medicine frequently encounter. We must also directly address unconscious biases that perpetuate less diverse environments. The Bias Reduction in Internal Medicine Program at the University of Wisconsin resulted in increased awareness, motivation, self-efficacy, and action for engaging in gender equity promoting activity, more positive departmental climate, and greater gender and race/ ethnicity diversity in new hires 2 to 3 years post-intervention (18).

Finally, it is our responsibility to use our collective voice as academic leaders in medicine and science to facilitate increas- ing URM students, trainees, and faculty in medicine and science. As recommended by past AAP President Dr. John Carethers, we can serve as mentors to URM individuals at any stage of training and invite URM students to participate in our research programs and clinical activities (19). Organized pipeline programs can help to facilitate this type of mentorship from high school through junior faculty level (20-22). The University of Pittsburgh Clinical and Translational Science Award partnered with 5 minority-serving institutions to create an intensive, one-year online training program for junior faculty and postdoctoral trainees to help early-career scientists launch successful biomedical research careers (22). At the end of the curriculum, which requires institutional protected time, participants bring a grant proposal and/or manuscript closer to fruition.

As outlined by Dr. Kieren Marr during her 2019 ASCI presidential address, less that $2 \%$ of the ASCI's membership are URM individuals and very few of these URMs are women. As medicine and science are evolving, it is important that we incorporate a diversity of backgrounds and scientific innovation. The ASCI might benefit from the model of the Robert Wood Johnson Harold Amos Faculty Development Program - a program created in 1983 to increase the number of faculty from historically disadvantaged backgrounds who can achieve senior rank in medicine, dentistry, or nursing (23). Following such an approach or engaging in a partnership could identify and mentor accomplished URM and URM women physician-scientists for future ASCI membership. The majority of our current URM ASCI members are former Harold Amos fellows, and this program has produced 
three recent National Institute of Health Institute Directors, three college/university Presidents, four Deans, and 10 Department Chairs. Finally, engaging URMs and women across the full spectrum of career stages, from student trainees to senior investigators, can help to champion solutions to increasing our diversity and ensuring further enhancements to our already impactful value.

Address correspondence to: Sherita Hill Golden, Hugh P. McCormick Family Professor of Endocrinology and Metabolism, Vice President, Chief Diversity Officer, Welch Center for Prevention, Epidemiology, and Clinical Research, Johns Hopkins Medicine, 1620 McElderry Street Street, Room 420, Baltimore, Maryland 21287, USA. Phone: 443.287.4827; Email: sahill@ jhmi.edu.

1. The National Academies Press. Sexual Harassment of Women: Climate, Culture, Consequences in Academic Sciences, Engineering, Medicine. Washington, DC, USA: National Academies Press; 2018.

2. Clancy KBH, Lee KMN, Rodgers EM, Richey C. Double jeopardy in astronomy and planetary science: Women of color face greater risks of gendered and racial harassment. J Geophys Res Planet. 2017;122(7):1610-1622.

3. Wasti SA, Cortina LM. Coping in context: sociocultural determinants of responses to sexual harassment. J Pers Soc Psychol. 2002;83(2):394-405.

4. Cortina LM, Fitzgerald LF, Drasgow F. Contextu- alizing Latina experiences of sexual harassment: preliminary tests of a structural model. Basic Appl Soc Psych. 2002;24(4):295-311.

5. Woods KC, Buchanan NT, Settles IH. Sexual harassment across the color line: experiences and outcomes of cross- versus intraracial sexual harassment among Black women. Cultur Divers Ethnic Minor Psychol. 2009;15(1):67-76.

6. Gomez LE, Bernet P. Diversity improves performance outcomes [published online ahead of print February 11, 2019]. J Natl Med Assoc. https://doi.org/10.1016/j.jnma.2019.01.006.

7. LaVeist TA, Pierre G. Integrating the 3Ds social determinants, health disparities, and health-care workforce diversity. Public Health Rep. 2014;129(suppl 2):9-14.

8. Pitts DW. Diversity, representation, and performance: evidence about race and ethnicity in Public organizations. Journal of Public Administration and Theory. 2005;15(4):615-631.

9. AlShebli BK, Rahwan T, Woon WL. The preeminence of ethnic diversity in scientific collaboration. Nat Commun. 2018;9(1):5163.

10. Duncan GA, et al. National Heart, Lung, and Blood Institute Workshop Summary: enhancing opportunities for training and retention of a diverse biomedical workforce. Ann Am Thorac Soc. 2016;13(4):562-567.

11. Heggeness ML, Evans L, Pohlhaus JR, Mills SL. Measuring diversity of the National Institutes of Health-funded workforce. Acad Med. 2016;91(8):1164-1172.

12. Mervis J. Mentoring's moment. Science. 2016;353(6303):980-982.

13. Lett LA, Orji WU, Sebro R. Declining racial and ethnic representation in clinical academic medicine: A longitudinal study of 16 US medical specialties. PLoS One. 2018;13(11):e0207274.

14. Harding CV, Akabas MH, Andersen OS. History and outcomes of 50 years of physician-scientist training in medical scientist training programs. Acad Med. 2017;92(10):1390-1398.

15. Wingard D, Trejo J, Gudea M, Goodman S, Reznik V. Faculty equity, diversity, culture and climate change in academic medicine: a longitudinal study. J Natl Med Assoc. 2019;111(1):46-53.

16. Golden SH, et al. A perspective on the Baltimore Freddie Gray riots: turning tragedy into civic engagement and culture change in an academic department of medicine. Acad Med 2018;93(12):1808-1813.

17. Wheeler DJ, Zapata J, Davis D, Chou C. Twelve tips for responding to microaggressions and overt discrimination: when the patient offends the learner. Med Teach. 2018:1-6.

18. Carnes M, et al. The effect of an intervention to break the gender bias habit for faculty at one institution: a cluster randomized, controlled trial. Acad Med. 2015;90(2):221-230.

19. Carethers JM. Facilitating minority medical education, research, and faculty. Dig Dis Sci. 2016;61(6):1436-1439.

20. Mains TE, Wilcox MV, Wright SM. Medical education resources initiative for teens program in baltimore: A model pipeline program built on four pillars. Educ Health (Abingdon). 2016;29(1):47-50.

21. Crockett ET. A research education program model to prepare a highly qualified workforce in biomedical and health-related research and increase diversity. BMC Med Educ. 2014;14:202

22. Rubio DM, et al. Developing a training program to diversify the biomedical research workforce [published online ahead of print February 12, 2019]. Acad Med. https://doi.org/10.1097/ ACM.0000000000002654.

23. Robert Wood Johnson Foundation. Harold Amos Medical Faculty Develoment Program 2019. RWJF website. http://www.amfdp.org/. Accessed June 25, 2019. 